\title{
Mesures de la taille et de la concentration de la suie dans une flamme laminaire prémélangée
}

\author{
R. Hadef1', V. Krüger ${ }^{2}$, K.P. Geigle ${ }^{3}$, M.S. Tsurikov ${ }^{3}$, Y. Schneider-Kühnle ${ }^{3}$ et M. Aigner ${ }^{3}$ \\ 1 BP 297 Institut de Génie Mécanique, Université Larbi Ben M’Hidi, 04000 Oum El Bouaghi - Algérie \\ 2 Robert Bosch GmbH, Corporate Research, Microsystem Technologies - Postfach 106050 - 70049 Stuttgart - Allemagne \\ 3 Institut für Verbrennungstechnik, DLR, Pfaffenwaldring 38-40, 70569 Stuttgart - Allemagne \\ e-mail : rhadef@rocketmail.com - veronique.krueger@de.bosch.com - klauspeter.geigle@dlr.de \\ michael.tsurikov@dlr.de - yorck.schneider-kuehnle@dlr.de - manfred.aigner@dlr.de
}

\begin{abstract}
Résumé - Une étude expérimentale est réalisée sur une série de flammes laminaires d'un prémélange propène-air. La flamme de forme conique est stabilisée sur un brûleur à surface poreuse et entourée par une flamme de méthane pour assurer son isolement. La technique d'incandescence induite par laser (IIL) calibrée par celle de l'extinction du laser, est utilisée pour déterminer la distribution spatiale (2D) de la fraction volumique de la suie pour trois valeurs de la pression, $P=1,3$ et 5 bar à diverses richesses. Pour les flammes à pression atmosphérique, l'investigation est étendue à la détermination de la taille des particules de suie par la mesure du taux de décroissance temporelle du signal IIL $(\mathrm{t})$. Pour ce dernier, la température locale des gaz est mesurée par la technique de diffusion Raman anti-Stokes cohérente (DRASC) décalée indispensable pour les flammes avec suie. Les résultats obtenus sont discutés et constituent une base de données pour valider d'éventuels résultats de modèles théoriques de simulation de la formation de la suie dans une flamme monodimensionnelle.
\end{abstract}

\begin{abstract}
Measurements of Soot Size and Concentration in the Laminar Premixed Flame - An experimental study has been carried out on several laminar premixed propene-air flames. The flame was stabilized in a dual-flame burner and a two-dimensional distribution of its soot volume fraction was measured using the laser induced incandescence (LII) technique for three values of the pressure: 1, 3 and 5 bar at various equivalence ratios. For the atmospheric flames, the investigation has been extended to evaluate a primary particle diameter from the measured temporal decay time of the LII signal. For this, the local temperature of gas has been measured by shifted vibrational coherent antiStokes Raman scattering, which yields well-resolved, accurate temperature measurements in sooting and non-sooting flames. The obtained results were discussed and should present a set of benchmark data for improvement and validation of numerical codes predicting the soot formation in a one-dimensional flame.
\end{abstract}




\section{INTRODUCTION}

De nos jours, les préoccupations profondes telles que la pollution atmosphérique et la surconsommation énergétique imposent aux ingénieurs chercheurs de développer des brûleurs propres et plus économiques. Leur conception exige préalablement une meilleure compréhension physico-chimique des processus de combustion qui est limitée par les expériences de diagnostics dans les foyers de combustion, qui n'atteignent pas la résolution de la modélisation numérique. Actuellement, seules les techniques basées sur les lasers pulsés et focalisés parviennent à surmonter ces limitations. De plus, le dernier développement technologique des lasers de puissance et des caméras CCD a rendu possible la combinaison quasi-simultanée de deux ou plusieurs techniques de diagnostics au dépend d'une augmentation des coûts [1]. Dans le cadre de développements récents menés au DLR (Deutsches Zentrum für Luft- und Raumfahrt), plusieurs techniques de diagnostics lasers ont été développés afin de disposer d'un panel unique de techniques directement applicables pour des écoulements réactifs similaires à ceux rencontrés dans des foyers industriels.

La présence de suie, produite généralement par les moteurs de véhicules terrestres et d'avions, est reconnue néfaste pour le rendement de la combustion, l'échauffement terrestre et la santé humaine [2-4]. Afin de développer des stratégies ou procédés pour réduire cette émission dangereuse, des études sur la suie sont exigées directement à la source de son émission c'est-à-dire dans les flammes de combustion. Elles sont menées dans deux directions : l'une consacrée au développement de théories de sa formation et l'autre sur les mesures expérimentales pour valider ces modèles.

Ce dernier point est l'objectif de cet article où la technique d'incandescence induite par laser, IIL [5] couplée à celle de l'extinction laser est appliquée pour mesurer la fraction volumique de la suie dans des flammes riches et laminaires d'un prémélange propène/air. Le choix du combustible est motivé d'une part par sa présence comme un intermédiaire important dans la combustion des alcanes forts, tels que le propane, le butane, l'heptane et l'iso-octane [6-9] et d'autre part par sa production d'espèces à forte concentration de radicaux $\mathrm{C}_{3}$ [9]. Comme la majorité des brûleurs industriels opèrent à haute pression, les mesures ont été réalisées pour trois valeurs de la pression 1, 3 et 5 bar. Pour les flammes atmosphériques, l'investigation expérimentale est étendue à la mesure de la température des gaz par la diffusion Raman antiStokes cohérente décalée (DRASC) [10] afin d'évaluer la taille moyenne des particules de suie à partir de la décroissance temporelle du signal IIL [11]. Cette technique, démontrée plus performante [12] que celle utilisant le rapport de l'intensité du signal IIL (intégrée dans le même espace temps) à deux longueurs d'onde de détection différentes [13], est largement employée dans les divers milieux de combustion et ne cesse de se développer considérablement [14-16].

\section{DISPOSITIF EXPÉRIMENTAL}

\subsection{Modèle du brûleur}

Le brûleur doit produire des flammes prémélangées laminaires et surtout stables à haute pression, afin de valider les modèles de simulation numérique en corrélant directement l'échelle de temps de réaction (et donc de la formation de la suie) avec la hauteur au-dessus du brûleur, HAB, dans des configurations unidimensionnelles. La flamme doit être donc homogène et large pour éviter les variations radiales de la grandeur physique à mesurer. Ainsi, la conception d'un tel brûleur est un défi technique de taille qui a pu être relevé dans cette étude en s'appuyant sur les réalisations antérieures des brûleurs à haute pression. Deux flammes concentriques et entourées par un écoulement d'air frais y sont stabilisées sur une surface poreuse de bronze (taille des micropores : $12 \mu \mathrm{m}$ ) refroidie par une circulation d'eau distillée régulée en température. La flamme annulaire $\mathrm{CH}_{4}$ /air (riche mais propre) protège la flamme centrale $\mathrm{C}_{3} \mathrm{H}_{6}$ /air à étudier contre les pertes thermiques latérales et donc réduit les effets de bord (oxydation). L'utilisation d'un écoulement d'azote pour entourer la flamme a été abandonnée parce qu'elle engendrait des dépôts polluants sur les fenêtres de visualisation et sur la soupape de commande de la pression dans la chambre de combustion [17]. Le diamètre du brûleur central est de 41,3 $\mathrm{mm}$. Les diamètres extérieurs du poreux de la flamme annulaire et de la conduite du co-écoulement d'air sont respectivement $61,3 \mathrm{~mm}$ et $150 \mathrm{~mm}$. L'ensemble du montage est installé dans une enceinte en acier refroidie par une circulation forcée d'eau et équipée de quatre fenêtres en quartz permettant l'accès optique. La pression à l'intérieur de la chambre de combustion est ajustée en variant les débits de gaz et/ou en fermant partiellement la conduite d'échappement des gaz brûlés avec un piston mobile commandé par un micro-ordinateur. La régulation du débit de chaque gaz est assurée par un débitmètre/

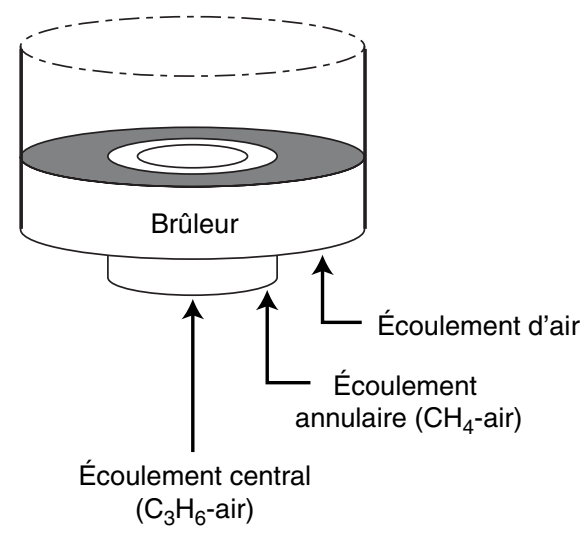

Figure 1

Schéma d'alimentation du brûleur en gaz. Schematic of the gas burner supply. 
régulateur volumique (Bronkhorst Hi-Tec., précision de $0,5 \%$, faible perte de charge et régulièrement calibré). La figure 1 présente un schéma simplifié de l'alimentation en gaz du brûleur.

\subsection{Diagnostics optiques}

\subsubsection{L'incandescence induite par laser}

Par absorption d'un puissant rayonnement laser pulsé (durée typique de $10 \mathrm{~ns}$ à $10 \mathrm{~Hz}$ ), les particules de carbone (suie) présentes dans une flamme s'échauffent rapidement jusqu'au voisinage de leur température de vaporisation puis refroidissent par différents modes de transferts thermiques (vaporisation, convection et rayonnement). L'émission radiative est aussi nommée incandescence. Durant tout ce processus, l'évolution temporelle du diamètre de la particule primaire, de la température de la suie, et par conséquent du signal IIL sont décrites par la conservation de son énergie et de sa masse dont les équations différentielles couplées sont résolues numériquement [18]. Il a été démontré que l'intensité maximale du signal d'incandescence émis est approximativement proportionnelle à la fraction volumique de la suie [5]. Cette linéarité a été confirmée par d'autres méthodes de mesure optique [19].

La figure 2 présente un exemple des évolutions temporelles du signal IIL calculées pour différents diamètres de la particule primaire. Il en ressort que plus la particule primaire est large, plus elle se refroidira lentement. La mesure de la décroissance temporelle du signal IIL est donc un indicateur sensible de taille.

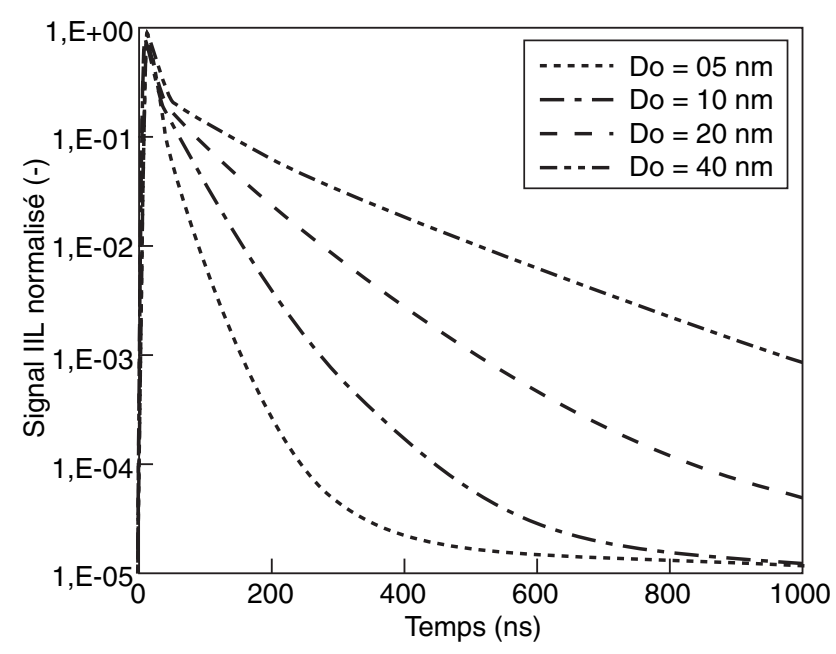

Figure 2

Simulation des évolutions temporelles du signal IIL pour différents diamètres de la particule primaire $\lambda_{\text {las }}=1064 \mathrm{~nm}$, densité du laser $0,6 \mathrm{~J} / \mathrm{cm}^{2}, \lambda_{\text {det }}=400 \pm 8 \mathrm{~nm}$.

Calculated time evolution of the LII signal for different primary particle sizes $\lambda_{\text {las }}=1064 \mathrm{~nm}$, laser fluence $0.6 \mathrm{~J} / \mathrm{cm}^{2}, \lambda_{\text {det }}=400 \pm 8 \mathrm{~nm}$.

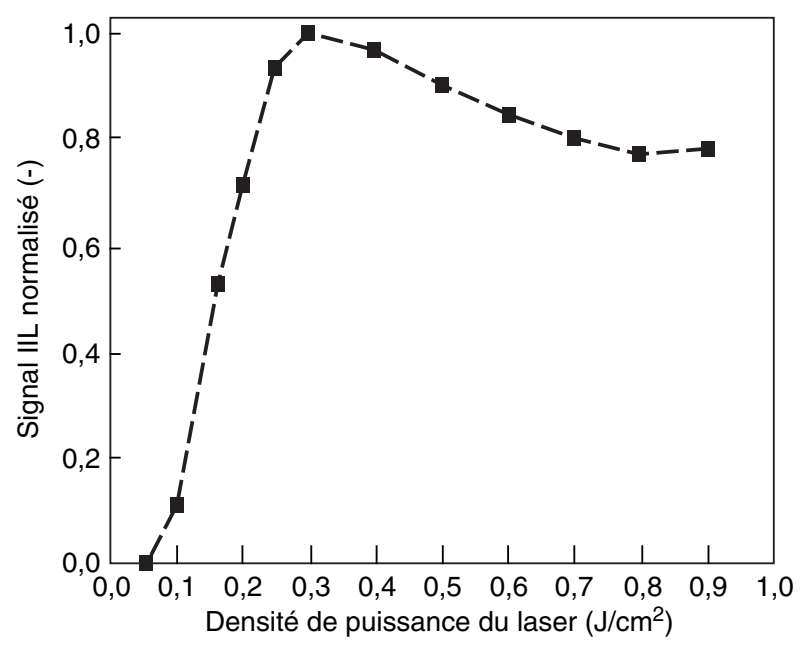

Figure 3

Variation du signal IIL en fonction de la puissance du laser. Variation of the LII signal with laser fluence.

Pour l'excitation, la longueur d'onde d'émission 1064 nm d'un laser Nd:YAG est sélectionnée pour éviter l'interférence avec la fluorescence des hydrocarbures polyaromatiques (HAP) et d'autres espèces émettant au voisinage de la fenêtre spectrale de détection (autour de $450 \mathrm{~nm}$ ). Le faisceau laser est ensuite élargi par une lentille cylindrique $(\mathrm{f}=-80 \mathrm{~mm})$ puis colmaté par une lentille sphérique ( $\mathrm{f}=100 \mathrm{~mm}$ ) pour former finalement un plan laser homogène de $170 \mu \mathrm{m}$ d'épaisseur. De cette bande, une portion de $30 \mathrm{~mm}$ de hauteur va exciter la flamme dans l'enceinte pressurisée. Ceci permet de délivrer une cartographie bidimensionnelle (image 2D) de la distribution en valeur relative de la suie dans la flamme. Un atténuateur (composé d'un polarisateur et d'une lame demi-onde qui fait tourner la polarisation) permet de régler la densité de puissance de la bande laser. Les mesures ont porté sur des flammes pouvant atteindre une concentration de la suie de l'ordre de $10 \mathrm{ppm}$ (à haute pression). Dans ce cas, l'absorption de l'intensité du laser à sa traversée de la flamme est très importante et pour pallier à ce handicap, sa puissance est réglée à la valeur voisine de $0,68 \mathrm{~J} / \mathrm{cm}^{2}$ (fig. 3). Pour la détermination de la taille de la suie, cette valeur est réduite à $0,5 \mathrm{~J} / \mathrm{cm}^{2}$ pour minimiser la sublimation.

L'émission incandescente de la suie est collectée perpendiculairement à la bande incidente après son passage à travers une combinaison de filtres $(448 \pm 15 \mathrm{~nm})$ par une caméra CCD intensifiée ( $8 \mathrm{pixel} / \mathrm{mm}$ ) avec deux images décalées dans le temps, chacune d'une durée de $40 \mathrm{~ns}$. La première, prise juste avant le pulse du laser (500 ns), permet de déterminer la luminosité de la flamme et la seconde est utilisée pour enregistrer le signal. Pour chaque mesure, l'image moyenne est réalisée sur une série de 100 prises consécutives. 


\subsubsection{L'extinction laser}

L'interprétation quantitative du signal IIL en fraction volumique des particules de suie nécessite la calibration en un point de la flamme, par une méthode complémentaire, souvent l'extinction laser. Dans ce cas, un faisceau laser de longueur d'onde $532 \mathrm{~nm}$ est utilisé. Il parcoure le même chemin optique que le faisceau de la IIL. Une partie est réfléchie directement sur une cellule colorant de référence (pour contrôler la variation temporelle de l'intensité du laser). L'autre partie traverse la chambre de combustion où le faisceau est partiellement absorbé par la flamme puis dirigé dans la seconde cellule colorant. Le rapport entre les signaux de fluorescence de la cuvette avec et sans flamme représente le coefficient de transmission. L'intensité du laser atténuée, $I$, détectée s'exprime le long du chemin d'extinction $L$ en fonction de l'intensité incidente $I_{0}$ selon la loi de Lambert-Beer :

$$
I=I_{0} \exp \left(-\int_{0}^{L} K_{e x} d l\right)
$$

Le coefficient d'extinction de la suie $K_{\text {ext }}$ est calculable par la théorie de Mie. Pour des petites particules sphériques, $\left(\pi D<<\lambda_{\text {ext }}\right)$ l'approximation de Rayleigh est valable et on a pour une distribution de taille des particules $P(D)$ :

$$
K_{e x t}=-\frac{\pi^{2}}{\lambda_{e x t}} \operatorname{Im}\left(\frac{\tilde{m}^{2}-1}{\tilde{m}^{2}+2}\right) N_{v} \int_{0}^{\infty} D^{3} P(D) d D
$$

où $\tilde{m}$ est l'indice (complexe) de réfraction de la suie à la longueur d'onde d'extinction $\lambda_{e x t}$ et $N_{v}$ est le nombre densité des particules. La fraction volumique des particules étant une combinaison du $N_{v}$ et $P(D)$ :

$$
f_{v}=\frac{\pi}{6} N_{v} \int_{0}^{\infty} D^{3} P(D) d D
$$

la combinaison des trois dernières équations aboutit à la fraction volumique moyenne de la suie :

$$
\overline{f_{v}}=-\frac{\lambda_{e x t} \ln \left(I / I_{0}\right)}{6 \pi L \operatorname{Im}\left(\frac{\tilde{m}^{2}-1}{\tilde{m}^{2}+2}\right)}
$$

La constante de calibration sera donc calculée par l'équation :

$$
C_{c a l}=-\frac{\lambda_{e x t} \ln \left(I / I_{0}\right)}{6 \pi L \operatorname{Im}\left(\frac{\tilde{m}^{2}-1}{\tilde{m}^{2}+2}\right) \int_{0}^{L} I_{I I L}(l) d l}
$$

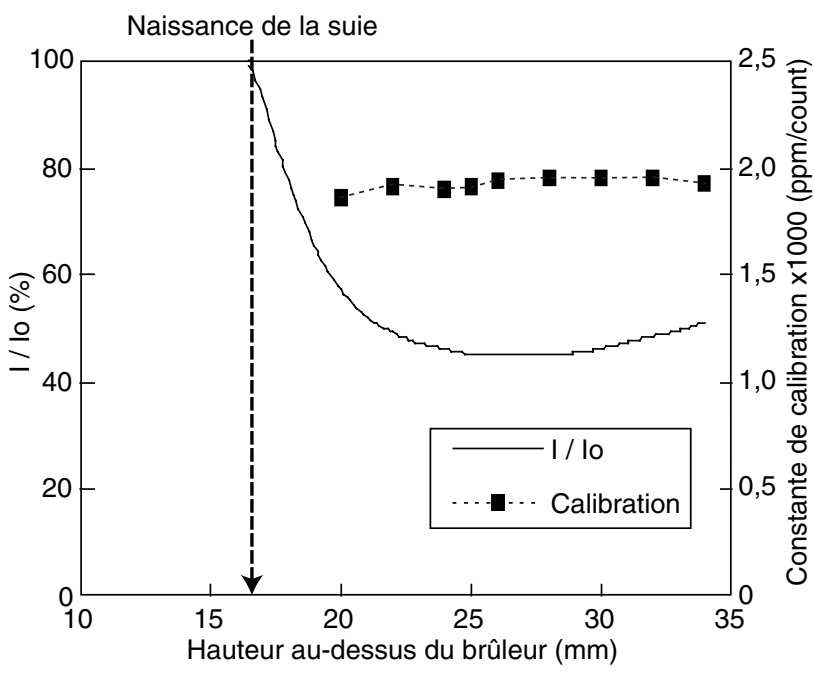

Figure 4

Profils axiaux du coefficient de transmission et de la constante de calibration le long de l'axe du brûleur $\left(\mathrm{C}_{3} \mathrm{H}_{6} / \mathrm{air}, 3\right.$ bar et $\phi=2,30)$.

Axial profiles of transmission and calibration constant along the burner axis $\left(\mathrm{C}_{3} \mathrm{H}_{6}\right.$ /air, 3 bar and $\left.\phi=2.30\right)$.

Dans cette étude, l'extinction est effectuée par une bande laser élargie à partir du faisceau et par conséquent, ce montage offre l'avantage de la position du point optimal de calibration après la mesure. La figure 4 présente un exemple des profils du rapport $I / I_{0}$ et de la constante de calibration mesurés le long de l'axe d'une flamme de propène à 3 bar. La calibration est faite pour chaque flamme (richesse et pression).

\subsubsection{La diffusion Raman anti-Stokes cohérente (DRASC)}

En dépit des difficultés significatives dans sa réalisation et l'interprétation de son signal, la DRASC s'est imposée comme une technique très efficace pour la mesure de température des gaz dans une large gamme de chambres de combustion. Son principe repose sur la focalisation au point de mesure de deux faisceaux de pompe de fréquence $\omega_{P}$ et un faisceau Stokes de fréquence $\omega_{S}$ modulable en fréquence, de sorte que la différence de fréquence $\omega_{P}-\omega_{S}$ soit égale à la fréquence Raman, $\omega_{R}$, que l'on veut exciter [20]. Cela provoque une interaction non linéaire des molécules avec les champs magnétiques intenses et génère un signal cohérent DRASC de fréquence $\omega_{D R A S C}=2 \omega_{P}-\omega_{S}$. Le faisceau Stokes et par conséquent le signal sont émis en bande large, ce qui permet d'enregistrer un spectre complet par point de mesure. La température est déterminée par fit des spectres rovibrationnels expérimentaux, de la branche $\mathrm{Q}$ de $\mathrm{N}_{2}$ (abondant dans les gaz de combustion) sur une bibliothèque de spectres DRASC calculés sur la base d'une distribution de Boltzmann aux différentes températures et la température produisant le meilleur ajustement de profils est prise comme température réelle. 


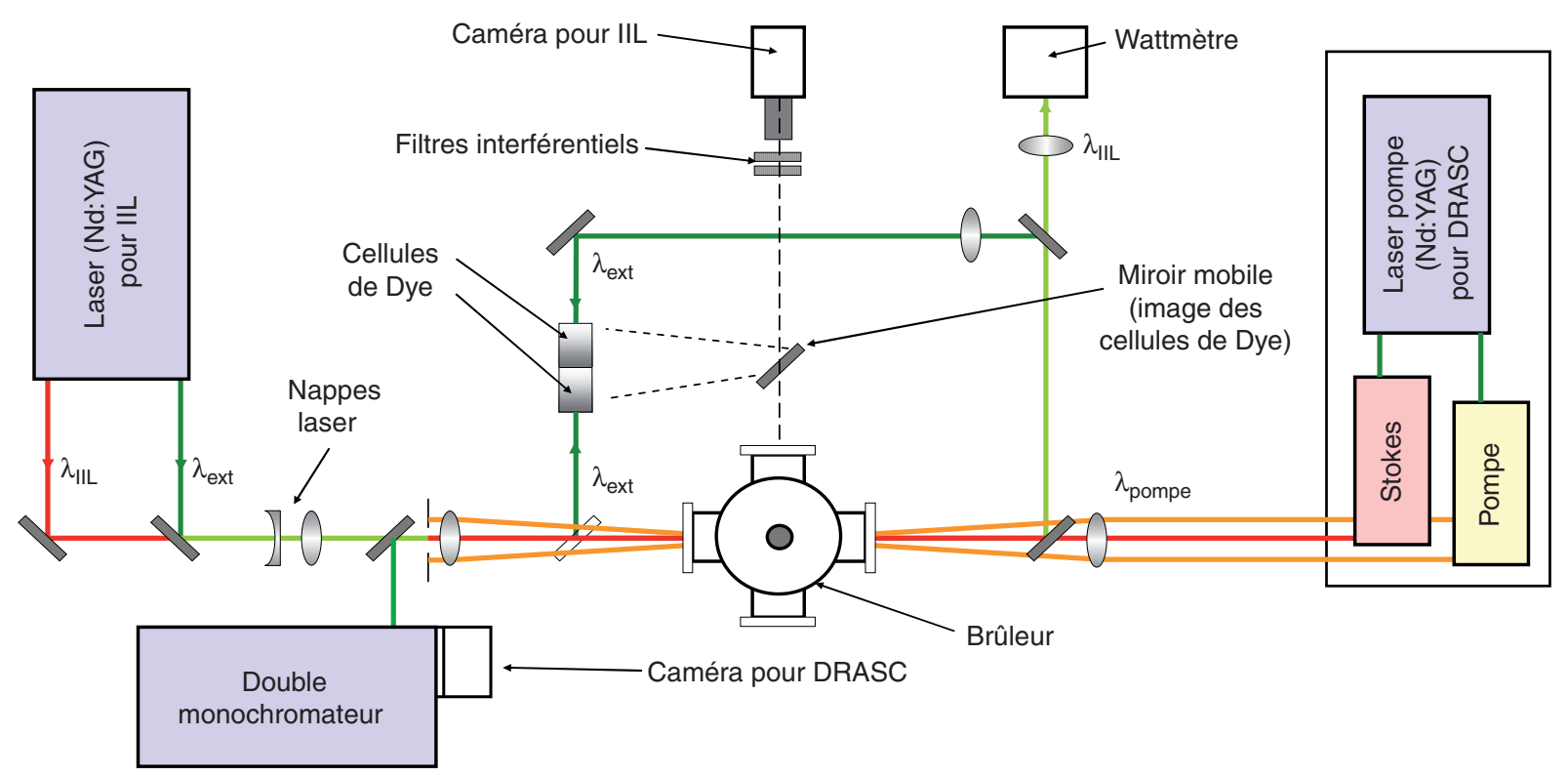

Figure 5

Schéma de l'ensemble du dispositif optique (IIL, extinction, DRASC) $\lambda_{\text {IIL }}=1064 \mathrm{~nm}, \lambda_{\text {ext }}=532 \mathrm{~nm}, \lambda$ (Stokes) $=685 \mathrm{~nm}$ et $\lambda$ (Pompe) $=591 \mathrm{~nm}$. Schematic of the optical set-up (LII, extinction, SV-CARS) $\lambda_{\text {LII }}=1064 \mathrm{~nm}, \lambda_{\text {ext }}=532 \mathrm{~nm}, \lambda($ Stokes $)=685 \mathrm{~nm}$ and $\lambda($ Pump $)=591 \mathrm{~nm}$.

TABLEAU 1

Caractéristiques et conditions opératoires des flammes étudiées Operating conditions for the flames studied

\begin{tabular}{|c|c|c|c|c|c|c|c|c|c|c|}
\hline \multirow{2}{*}{$\begin{array}{c}\mathrm{P} \\
\text { (bar) }\end{array}$} & \multicolumn{4}{|c|}{$\begin{array}{l}\text { Flamme centrale (suitée) } \\
\qquad \mathrm{C}_{3} \mathrm{H}_{6} / \text { air }\end{array}$} & \multicolumn{4}{|c|}{$\begin{array}{l}\text { Flamme annulaire (propre) } \\
\qquad \mathrm{CH}_{4} / \text { air }\end{array}$} & \multicolumn{2}{|c|}{ Co-écoulement d'air } \\
\hline & $\begin{array}{l}\Phi \\
(-)\end{array}$ & $\begin{array}{c}\mathrm{Q}_{\text {fuel }} \\
([\mathrm{ln} / \mathrm{mn})\end{array}$ & $\begin{array}{c}\mathrm{Q}_{\mathrm{air}} \\
(\mathrm{ln} / \mathrm{mn})\end{array}$ & $\begin{array}{c}\mathrm{U} \\
(\mathrm{cm} / \mathrm{s})\end{array}$ & $\begin{array}{l}\Phi \\
(-)\end{array}$ & $\begin{array}{c}\mathrm{Q}_{\text {fuel }} \\
(\mathrm{ln} / \mathrm{mn})\end{array}$ & $\begin{array}{c}\mathrm{Q}_{\text {air }} \\
(\ln / \mathrm{mn})\end{array}$ & $\begin{array}{c}\mathrm{U} \\
(\mathrm{cm} / \mathrm{s})\end{array}$ & $\begin{array}{c}\mathrm{Q}_{\mathrm{air}} \\
(\ln / \mathrm{mn})\end{array}$ & $\begin{array}{c}\mathrm{U} \\
(\mathrm{cm} / \mathrm{s})\end{array}$ \\
\hline \multirow{2}{*}{1} & 2,23 & 0,38 & 3,60 & 5,44 & 1,31 & 1,63 & 11,94 & 15,42 & 182,4 & 22,69 \\
\hline & 2,46 & 0,41 & 3,60 & 5,48 & 1,36 & 1,68 & 11,82 & 15,34 & 182,4 & 22,69 \\
\hline \multirow{2}{*}{3} & 2,10 & 0,83 & 8,43 & 4,21 & 1,35 & 5,38 & 37,96 & 16,40 & 416,2 & 17,25 \\
\hline & 2,30 & 0,90 & 8,43 & 4,25 & 1,35 & 5,38 & 37,96 & 16,40 & 416,2 & 17,25 \\
\hline \multirow{4}{*}{5} & 1,95 & 0,92 & 10,2 & 3,04 & 1,40 & 8,92 & 61,0 & 15,90 & 462 & 11,49 \\
\hline & 2,05 & 0,97 & 10,2 & 3,05 & 1,40 & 8,97 & 61,1 & 15,9 & 462 & 11,49 \\
\hline & 2,15 & 1,02 & 10,2 & 3,06 & 1,40 & 8,97 & 61,1 & 15,91 & 462 & 11,49 \\
\hline & 2,30 & 0,89 & 8,37 & 2,53 & 1,40 & 7,71 & 52,41 & 13,65 & 416 & 10,34 \\
\hline
\end{tabular}

Généralement, le système classique de DRASC pour déterminer la température des gaz dans une flamme utilise les faisceaux de pompe à $532 \mathrm{~nm}$ et un faisceau de Stokes centré à $607 \mathrm{~nm}$. En présence de suie, la bande spectrale d'émission (468-475 $\mathrm{nm}$ ) de la fluorescence des radicaux $\mathrm{C}_{2}$ induite par laser [21] coïncide avec le spectre d'émission du signal $\mathrm{N}_{2}$-DRASC (max. à 473,5 nm). Pour pallier cette limitation, les longueurs d'onde d'excitation sont modifiées de sorte que le signal DRASC de $\mathrm{N}_{2}$ soit décalé dans une autre région spectrale plus adéquate. Dans la présente configuration, la longueur d'onde $532 \mathrm{~nm}$ est utilisée pour pomper un laser colorant à bande étroite qui fournit deux faisceaux pompes $(591 \mathrm{~nm})$ et un laser colorant à large bande qui fournit le faisceau Stokes $(685 \mathrm{~nm})$. Le signal de DRASC se trouve donc décalé spectralement de la longueur d'onde $473 \mathrm{~nm}$ à $520 \mathrm{~nm}$ où il peut être aisément détecté sans interférences [10]. Les mesures de température obtenues par cette variante de DRASC dans une flamme propre de diffusion corroborent celles de la DRASC conventionnelle avec une incertitude de $\pm 3 \%$ [22]. L'ensemble du dispositif optique est schématisé par la figure 5. 


\section{RÉSULTATS}

Compte tenu de la structure dynamique de l'écoulement résultant de l'interaction entre les différentes vitesses des écoulements gazeux à leur sortie du brûleur, l'obtention d'une flamme stable n'était pas systématique surtout pour les flammes à haute pression. Une étude préalable pour trouver des conditions adéquates était nécessaire. Le tableau 1 présente l'ensemble des flammes sélectionnées pour cette étude avec leurs caractéristiques.

\subsection{Flammes atmosphériques}

Pour plus de clarté, la température des gaz, $T_{g}$ et la fraction volumique de la suie, $\mathrm{f}_{\mathrm{v}}$, mesurées le long de l'axe de la flamme sont représentées en fonction de HAB, dans la même figure (fig. 6). Suite au dégagement énergétique des réactions exothermiques, la température des gaz augmente fortement dans la zone de préchauffage pour atteindre dans la zone de réaction un maximum très proche de la température adiabatique théorique $\left(T_{\max }=1665 \pm 49 \mathrm{~K}\right.$ pour $T_{\text {adiab }}=$ $1670 \mathrm{~K}$ et $T_{\text {max }}=1560 \pm 47 \mathrm{~K}$ pour $T_{\text {adiab }}=1545 \mathrm{~K}$ ). Rappelons que dans la zone de réaction la concentration en oxygène est quasiment nulle. Plusieurs espèces intermédiaires, tels que $\mathrm{C}_{2} \mathrm{H}_{2}, \mathrm{C}_{3} \mathrm{H}_{4}$, et $\mathrm{C}_{6} \mathrm{H}_{6}$ sont produites dans ces réactions exothermiques. Dans la zone de post-combustion, la température des gaz décroît progressivement à cause des radiations thermiques de la zone lumineuse et des réactions endothermiques responsables de la formation de la suie. Cette structure est fondamentalement commune aux autres flammes. A cause de sa haute vitesse, le front de la flamme

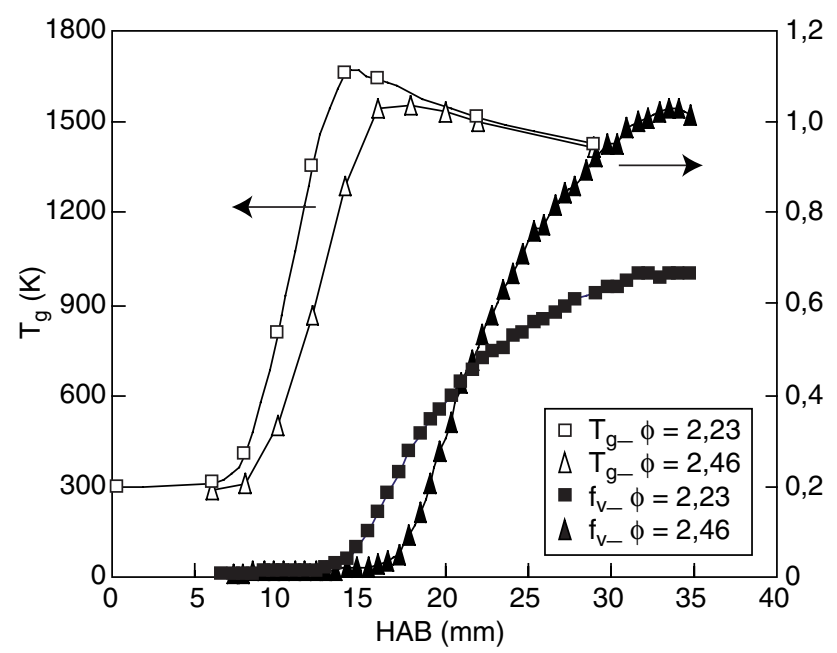

Figure 6

Température des gaz et fraction volumique de la suie le long de l'axe de la flamme $\left(\mathrm{C}_{3} \mathrm{H}_{6}, 1\right.$ bar $)$.

Gas temperature and soot volume fraction along the flame axis $\left(\mathrm{C}_{3} \mathrm{H}_{6}, 1\right.$ bar $)$. moins riche est positionné plus proche de la surface du brûleur que celui de la flamme plus riche. En revanche, la flamme de richesse 2,46 a une vitesse plus petite, et se trouve donc stabilisée plus loin de la surface du brûleur. Malgré leurs richesses relativement proches, la différence entre les maximums de températures est significative : de l'ordre de $100 \mathrm{~K}$. La température affecte les deux processus compétitifs présents dans les flammes prémélangées suitées : le taux de pyrolyse des espèces intermédiaires menant aux précurseurs et à l'attaque oxydante (radical $\mathrm{OH}$ ) sur ces précurseurs [23]. Comme l'accroissement du second taux est supérieur à celui du premier, la flamme moins riche produit moins de suie à cause de sa température élevée. D'un autre côté, la concentration des espèces aromatiques croît avec la richesse. Il en découle une augmentation de la production de suie, puisque les énergies d'activation apparentes des réactions de croissance de surface sont très faibles. La concentration de suie augmente de façon exponentielle avec la hauteur au-dessus du brûleur. Cette augmentation correspond à la formation de la suie dans la flamme. Dans la phase finale du processus de combustion, $f_{v}$ atteint une valeur finale presque constante bien que la concentration de $\mathrm{C}_{2} \mathrm{H}_{2}$ reconnu comme représentatif de la croissance des espèces soit élevée dans les gaz brûlés [24]. Ceci est attribué à la décroissance de l'activité des particules de suie résultant de la réduction de la concentration de $\mathrm{H}$ quand la température décroît $[25,26]$. Ces mécanismes ont été expliqués par des modèles incorporant les concepts des processus d'abstraction d'hydrogène - addition du carbone (AHAC) [27, 28].

L'image de l'intensité du signal IIL enregistrée par la caméra CCD (fig. 7a) est d'abord filtrée avec l'image de la luminosité de la flamme. L'image obtenue est ensuite traitée pour reconstituer la distribution bidimensionnelle de la fraction volumique de la suie (fig. $7 b$ ) à l'aide de la constante de calibration et d'un algorithme de décomposition (onion-peel) qui tient compte de l'absorption du signal IIL par la suie avant d'atteindre la caméra [29]. Ce traitement est effectué pour chaque point de mesure (pixel). Qualitativement, la répartition $2 \mathrm{D}$ de la concentration de la suie est identique pour les deux flammes. Le profil radial de $f_{v}$ est quasi-uniforme dans la partie centrale de chaque flamme indépendamment de $\mathrm{HAB}$, confirmant la non interaction de cette zone avec la flamme annulaire. Sur les bords de cette dernière, la fraction volumique de suie décroît rapidement par oxydation et par manque de combustible jusqu'à la valeur zéro.

L'évaluation du diamètre moyen de la particule de suie à partir de la mesure du taux de décroissance du signal IIL(t), dépend fortement de l'expression du terme de déperditions convectives, $\dot{Q}_{\text {conv }}$, de la température des gaz et du coefficient d'accommodation thermique, $\alpha_{T}$ [30]. Dans le cas présent, le modèle de base [5] est utilisé dans une version incluant en plus de la valeur exacte de la température locale des gaz mesurée par la DRASC, les récents améliorations et développements de la théorie de IIL tels que les valeurs 


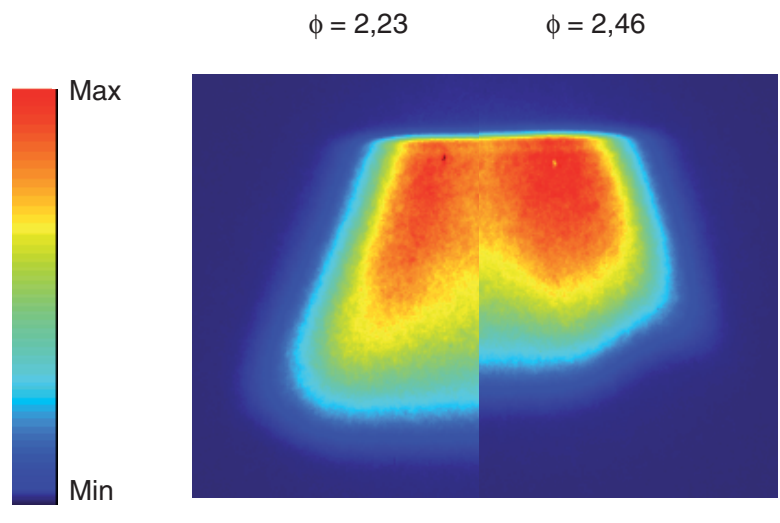

a)

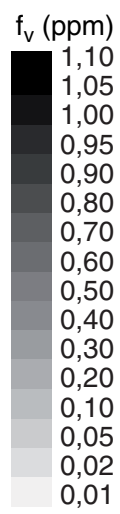

b)

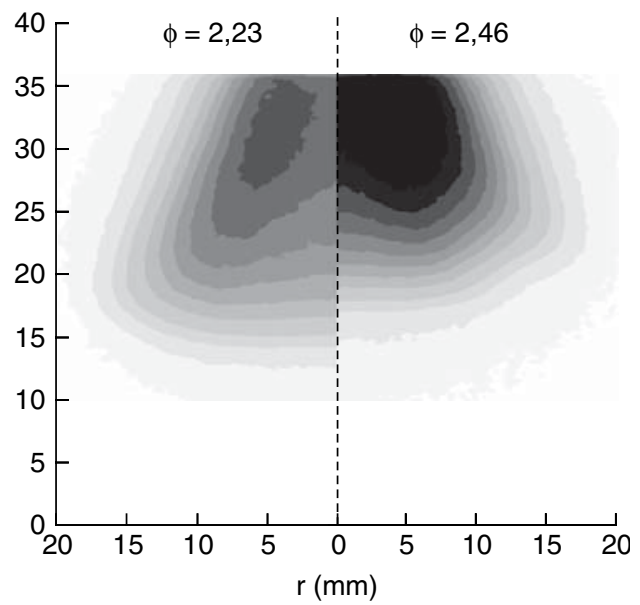

Figure 7

Distribution spatiale du signal IIL a) et de la fraction volumique de la suie b) en ppm. Spatial distribution of the LII signal (a) and soot volume fraction (b) in ppm.

TABLEAU 2

Différences entre le modèle original [5] et celui employé dans la présente étude Differences between the initial model [5] and the model used in this study

\begin{tabular}{|c|c|c|}
\hline & Modèle de Melton & Présent modèle \\
\hline Énergie Interne & $\rho_{s}$ et $C_{s}:$ constantes & $\rho_{s}, C_{s}$ dépendant de la température de la suie \\
\hline Absorption & constante & $\alpha=4 \pi D E_{\lambda} / \lambda$ \\
\hline Radiation & constante & $\varepsilon=4 \pi D E_{\lambda} / \lambda$ \\
\hline Sublimation & $\begin{array}{l}\text { - Vitesse de la vapeur de suie: } \\
U_{v}=\left(R T_{s} / 2 M_{v}\right)^{0,5} \\
\text { - } M_{v}, \Delta H_{v}, P_{v}: \text { constantes }\end{array}$ & $\begin{array}{l}\text { - Vitesse de la vapeur de suie : } \\
U_{v}=0,8\left(R T_{s} / 2 \pi M_{v}\right)^{0,4} \\
\text { - } M_{v}, \Delta H_{v}, P_{v} \text { dépendant de la température de la suie }\end{array}$ \\
\hline Conduction & $\begin{array}{l}\text { Régime de transition avec un coefficient } \\
\text { d'accommodation thermique } \alpha_{T}=0.9\end{array}$ & $\begin{array}{l}\text { Régime libre si } K_{n}>5 \sqrt{\gamma \pi / 2} \\
\text { Régime de transition si } K_{n}<5 \sqrt{\gamma \pi / 2} \\
\alpha_{T}=0.3 \text { (dans les deux cas) }\end{array}$ \\
\hline
\end{tabular}

mesurées de la fonction d'absorption de la suie $E(m)$ et de $\alpha_{T}$ [31], la prise en compte de la variation avec la température des propriétés thermo-physiques de la suie (masse volumique, chaleur massique, enthalpie d'évaporation, masse molaire et pression d'évaporation) $[32,33]$, l'expression de $\dot{Q}_{\text {conv }}$ propre à chaque régime (continu et libre) [34] et enfin la valeur de 0,4 au lieu de 0,5 pour l'exposant $n$ apparaissant dans l'expression de la vitesse des molécules de vapeur résultant de l'évaporation de la suie [35]. Dans cette étude, la distribution de la taille des particules est supposée de type lognormal avec une déviation standard $\ln (\sigma)=0,34$ [36]. Le tableau 2 présente les différences entre les deux modèles.

La performance de l'actuel modèle est validée par les mesures de température de la particule de suie d'une flamme de diffusion d'éthane à partir d'un lissage des spectres d'émission de Planck mesurés [37] (le type de flamme, de prémélange ou de diffusion, n'intervient pas dans la formulation théorique de la résolution temporelle du signal IIL). Hormis la phase d'évaporation dont la modélisation demeure toujours complexe, la décroissance temporelle de la température est bien prédite par ce modèle pour une valeur du diamètre initial de $25 \mathrm{~nm}$ mesuré sur l'axe de la flamme à une distance $\mathrm{HAB}=20 \mathrm{~mm}$ (fig. 8).

Pour la détermination du diamètre moyen de la particule et contrairement à la méthode utilisant le rapport de IIL(t) à deux instants de mesure décalés, notre analyse 2D exploite le signal IIL(t) à plusieurs instants de mesure pour un lissage de sa décroissance temporelle à partir de son maximum par 

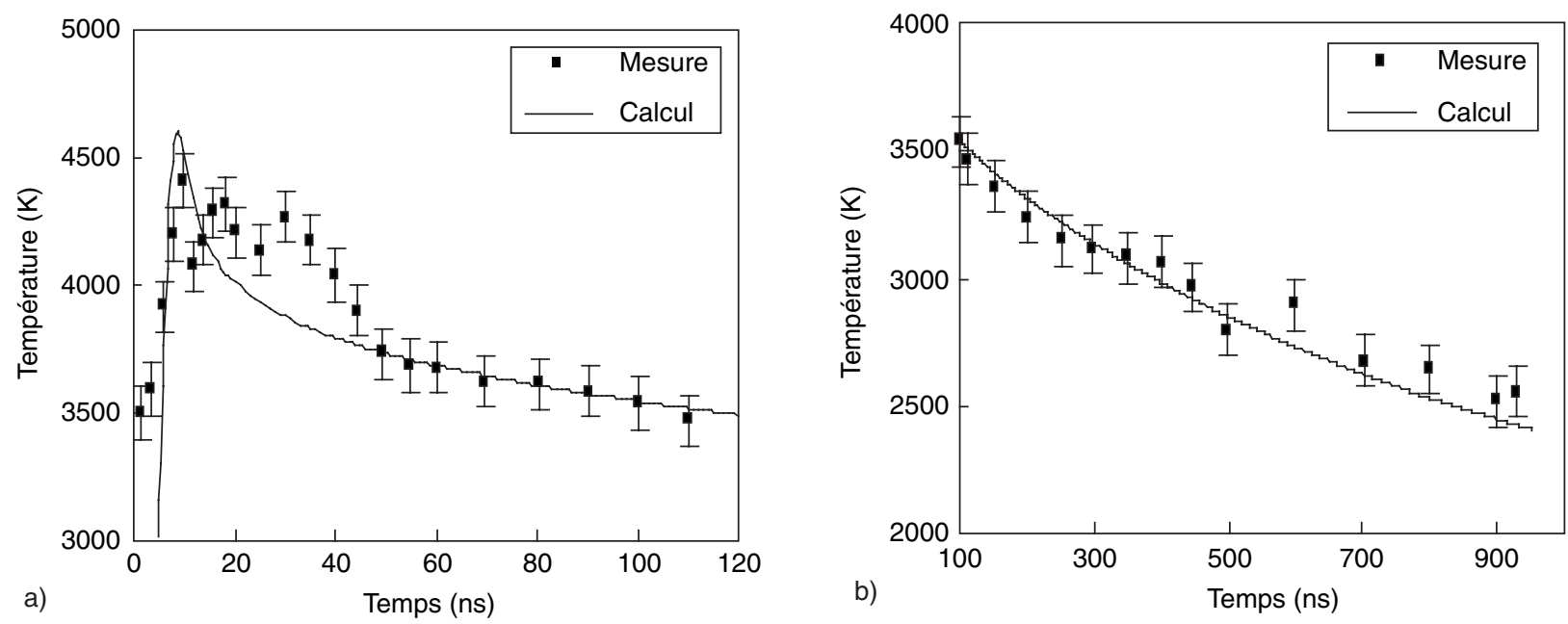

Figure 8

Evolution temporelle de la température de la particule de suie de diamètre primaire $25 \mathrm{~nm}$ dans une flamme de diffusion d'éthane (mesurée [37] et calculée par le présent modèle).

Time evolution of the temperature of a $25 \mathrm{~nm}$ diameter primary soot particle in an ethane diffusion flame (measured [37] and calculated using the present model).

une fonction mathématique. Une double exponentielle $\mathrm{A} \exp \left(-\mathrm{t} / \tau_{1}\right)+\mathrm{B} \exp \left(-\mathrm{t} / \tau_{2}\right)$ décrit mieux la décroissance du signal IIL qu'une simple, à cause de la présence de la vaporisation pendant les premières $50 \mathrm{~ns}$ (fig. 9). Les valeurs obtenues dans une flamme d'éthylène de diffusion, par cette méthode et celle mesurée directement par microscopie électronique à transmission (TEM) présentent une incertitude de $9 \%$ pour des stations $\mathrm{HAB}<50 \mathrm{~mm}$ et de l'ordre de $25 \%$ au-delà [12].

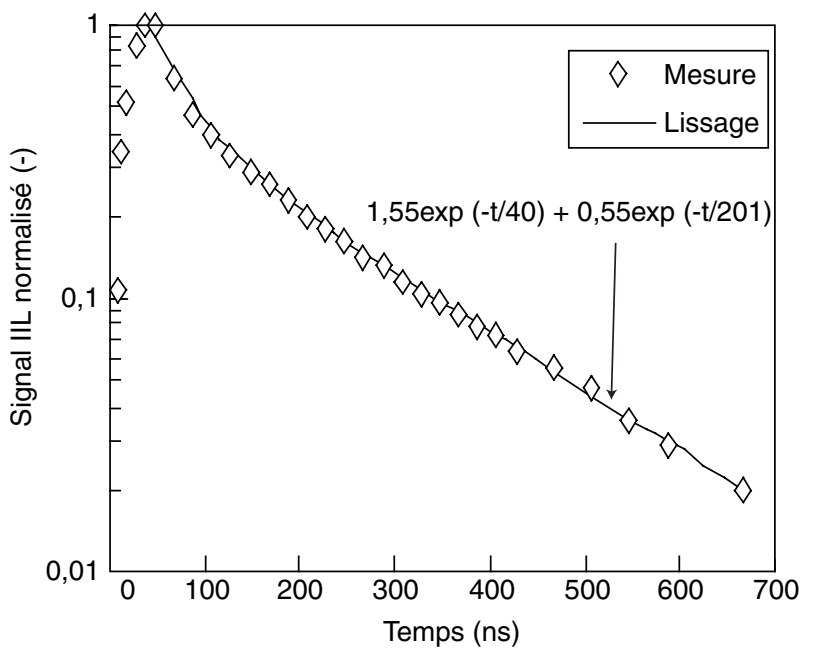

Figure 9

Evolution temporelle du signal IIL prise par la caméra. Temporal characteristics of the LII signal captured by the camera.
Puisque les mesures des fluctuations temporelles de l'intensité du signal IIL (non présentées ici), confirment la stabilité de la flamme, l'effet du décalage temporel de l'acquisition par la caméra est négligeable. Pour l'interprétation du signal, le temps de décroissance court ne montre aucune dépendance de la taille de la particule avec HAB. En revanche, le temps de décroissance lent est utilisé pour la mesure du diamètre moyen de la particule. Ce dernier croit rapidement avec HAB à cause de la coagulation et atteint en aval une limite finie dont la valeur croit avec la richesse (fig. 10a). Aux bords de la flamme, l'oxydation réduit la taille de ces particules (fig. 10b). A chaque hauteur, l'évolution radiale du diamètre moyen de la particule de suie primaire est semblable à celle de la fraction volumique de la suie (fig. 11).

La croissance de $f_{v}$ est analysée en fonction de son temps de résidence dans la flamme. Le résultat obtenu (fig. 12a) est une relation phénoménologique de $d f_{v} / d t=-k_{c}\left(f_{v}-f_{v, \text { max }}\right)$ linéarité où $k_{c}$ est un coefficient dépendant uniquement de la température maximale [38] et interprété comme une mesure effective du temps de vie active des particules avant de perdre leur réactivité $[27,39]$. À chaque point de mesure, la valeur de $d f_{v} / d t$ est calculée à partir de la fonction lissant sept points de mesure centrés sur ce point. Pour plus d'informations, cette analyse a été étendue à deux autres flammes (éthylène et toluène pré-évaporé) obtenues dans les mêmes conditions expérimentales [40]. L'interprétation des résultats révèle une variation de type Arrhenius : $k_{\mathrm{c}} \propto \exp \left(-E_{a} / R T_{\text {max }}\right)$ avec une valeur de l'énergie d'activation apparente $E_{a}=15 \mathrm{kcal} / \mathrm{mol}$ rapportée au temps de résidence (fig. 12b). 




Figure 10a

Diamètre moyen de la particule de suie le long de l'axe de la flamme $\left(\mathrm{C}_{3} \mathrm{H}_{6}, 1\right.$ bar $)$.

Mean soot particle diameter along the flame axis $\left(\mathrm{C}_{3} \mathrm{H}_{6}\right.$, 1 bar).

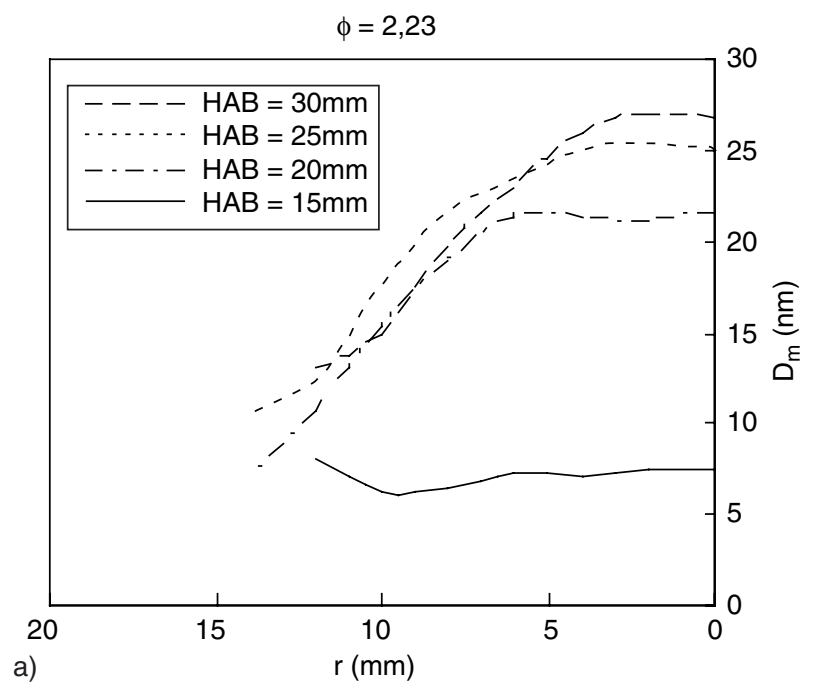

Figure 11

Profils radiaux du diamètre moyen de la particule de suie $\left(\mathrm{C}_{3} \mathrm{H}_{6}, 1\right.$ bar). Radial profile of the mean soot particle diameter $\left(\mathrm{C}_{3} \mathrm{H}_{6}, 1 \mathrm{bar}\right)$.

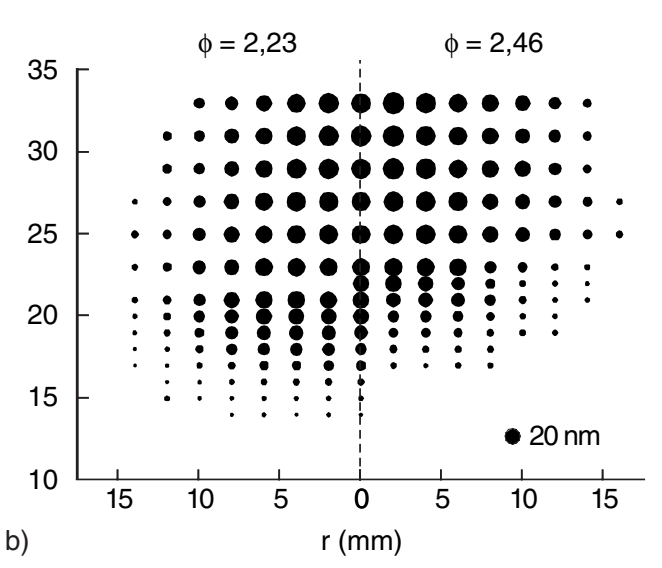

Figure 10b

Distribution 2D du diamètre moyen de la particule de suie $\left(\mathrm{C}_{3} \mathrm{H}_{6}, 1\right.$ bar $)$.

$2 D$ distribution of the mean soot particle diameter $\left(C_{3} H_{6}\right.$, 1 bar).

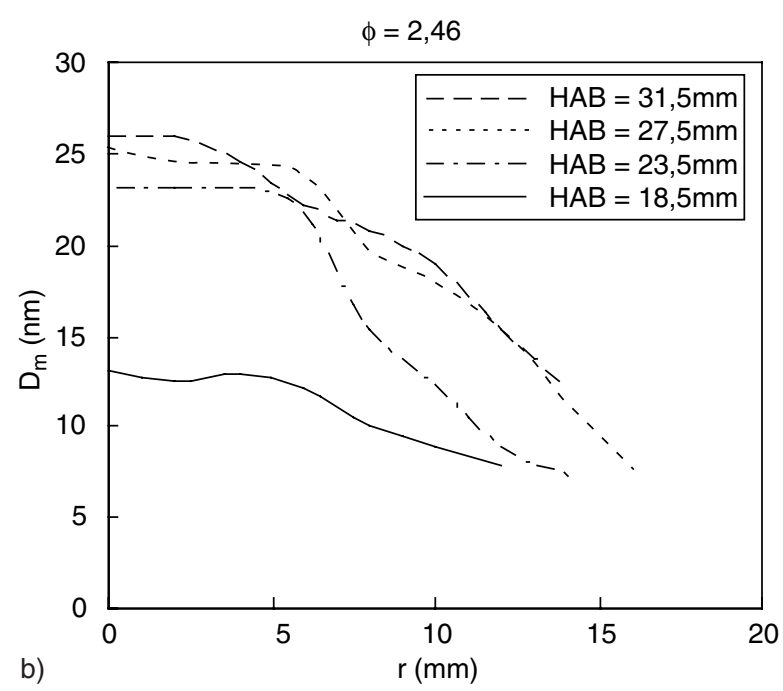

\subsection{Flammes à haute pression}

La figure 13 montre l'augmentation de la quantité de suie formée avec la pression. Cette croissance est corrélée à la densité du mélange gazeux et au temps de résidence. Le processus de croissance de la suie s'arrête suite au manque de $\mathrm{C}_{2} \mathrm{H}_{2}$ dans les gaz brûlés contrairement à la flamme atmosphérique [41]. La structure 2D de la distribution de la suie présentée en figure 14 pour deux valeurs de la pression 3 et
5 bar, est similaire à celle de 1 bar si ce n'est l'apparition sur les bords de la flamme d'une zone plus suitée. Cela correspond à une structure de flamme de diffusion créée par l'interaction des produits de combustion non-brûlés de la flamme annulaire avec l'air qui l'entoure. L'écoulement gazeux étant intrinsèquement bidimensionnel dans cette région, les caractéristiques de la suie (formation, croissance et oxydation) y sont plus complexes [42]. 


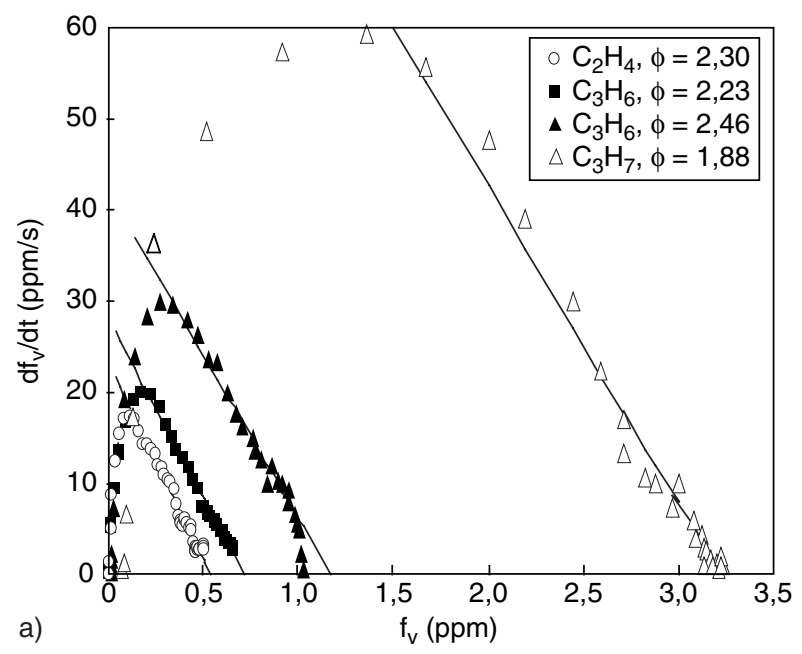

Figure 12a

Variation de $d f_{v} / d t$ en fonction de $f_{v}$ pour différents combustibles mesurées à 1 bar.

Variation of $d f_{v} / d t$ as a function of $f_{v}$ for different fuels measured at 1 bar.

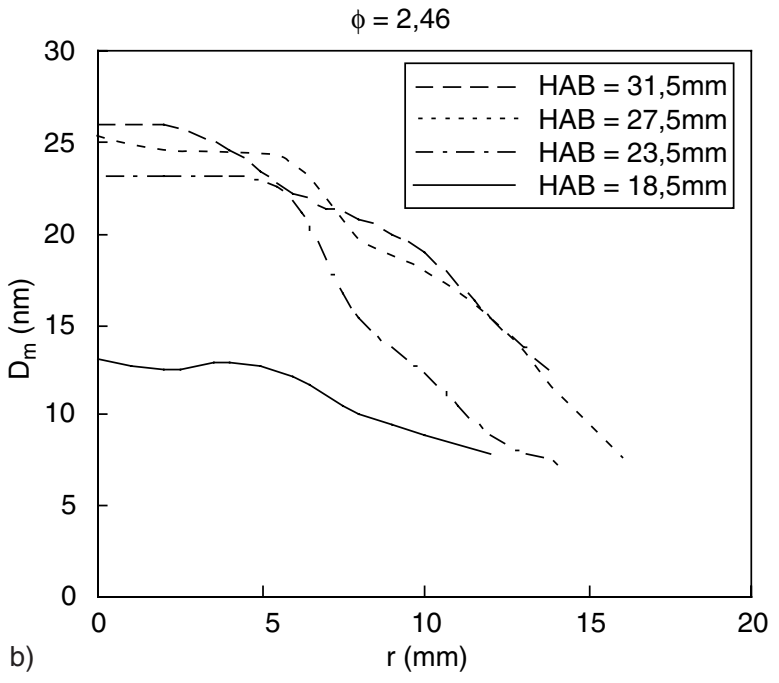

Figure 12b

Variation de $k_{c}$ en fonction de $T_{\max }$ pour différents combustibles mesurées à 1 bar.

Variation of $k_{c}$ as a function of $T_{\max }$ for different fuels measured at 1 bar.

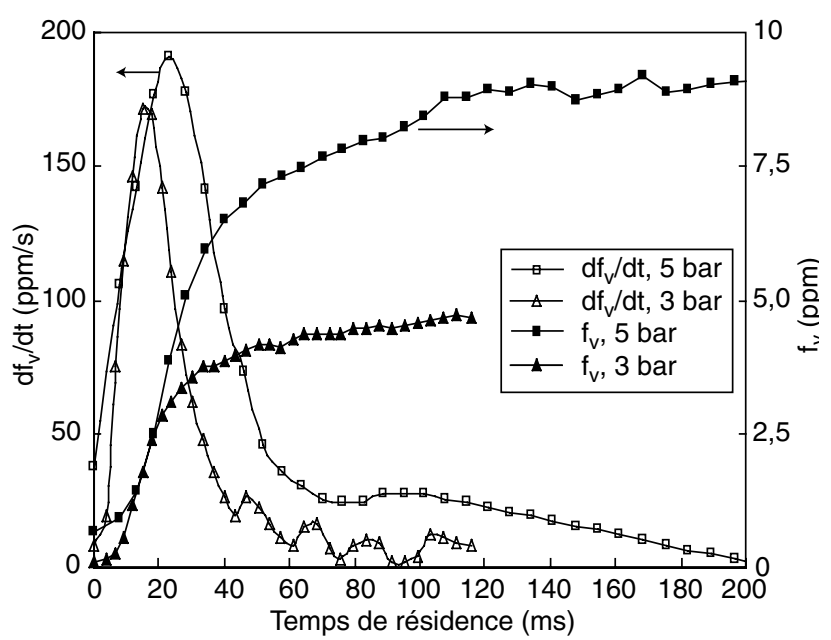

Figure 13

$d f_{v} / d t$ et $f_{v}$ le long de l'axe de la flamme $\left(\mathrm{C}_{3} \mathrm{H}_{6}, \phi=2.30\right)$ en fonction du temps de résidence.

$d f_{v} / d t$ and $f_{v}$ along the flame axis $\left(C_{3} H_{6}, \phi=2.30\right)$ as a function of residence time

La figure 15a révèle une croissance linéaire de la valeur maximale de la fraction volumique de la suie, $f_{v, \max }$ avec la richesse pour une pression de 5 bar. Cette propriété était déjà connue pour la flamme atmosphérique d'éthylène [43]. Sur la figure $15 \mathrm{~b}$, on peut observer une dépendance non linéaire d'ordre 1.5 de $f_{v, \text { max }}$ avec la pression alors qu'il a été trouvé que $f_{v, \text { max }} \propto P^{2}$ pour la flamme plane $\mathrm{C}_{2} \mathrm{H}_{4} /$ air [44] en variant uniquement la pression jusqu'à 10 bar. Au-delà de cette valeur le taux de croissance de la suie est moins sensible à la pression $f_{v, \text { max }} \propto P$ [42]. Dans notre étude, en plus de la pression, les flammes diffèrent par les conditions dynamiques de l'écoulement (vitesse, température de flamme) donc du temps de résidence. Ceci justifie l'écart d'exposant entre les deux lois. D'un autre côté, pour la flamme de diffusion, on a obtenu une loi de $f_{v, \text { max }} \propto P^{3}$ [45]. Notons que la valeur de $f_{v, \text { max }}$ pour la richesse de 2.30 à la pression atmosphérique a été interpolée linéairement entre les richesses 2.23 et 2.46 [43].

\section{CONCLUSION}

La technique d'incandescence induite par laser (IIL) a été utilisée pour mesurer la distribution bidimensionnelle de la suie dans une flamme laminaire d'un prémélange propène-air, en fonction de la richesse et de la pression. L'allure du profil de la fraction volumique de la suie le long de l'axe de la flamme est similaire pour toutes les flammes. L'augmentation de la quantité de la suie avec la richesse est mise en évidence pour trois valeurs de la pression 1, 3 et 5 bar. Les profils radiaux de fraction volumique de la suie, $\mathrm{f}_{\mathrm{v}}$ mesurés à différentes valeurs de la hauteur au-dessus du brûleur présentent indépendamment de la pression une région centrale où $\mathrm{f}_{\mathrm{v}}$ et le diamètre moyen de la particule de suie primaire sont uniformes. Par conséquent, les résultats obtenus le long de l'axe du brûleur constituent un outil de validation des modèles théoriques simulant la formation de la suie dans des flammes planes. La température locale des gaz est mesurée par la diffusion 

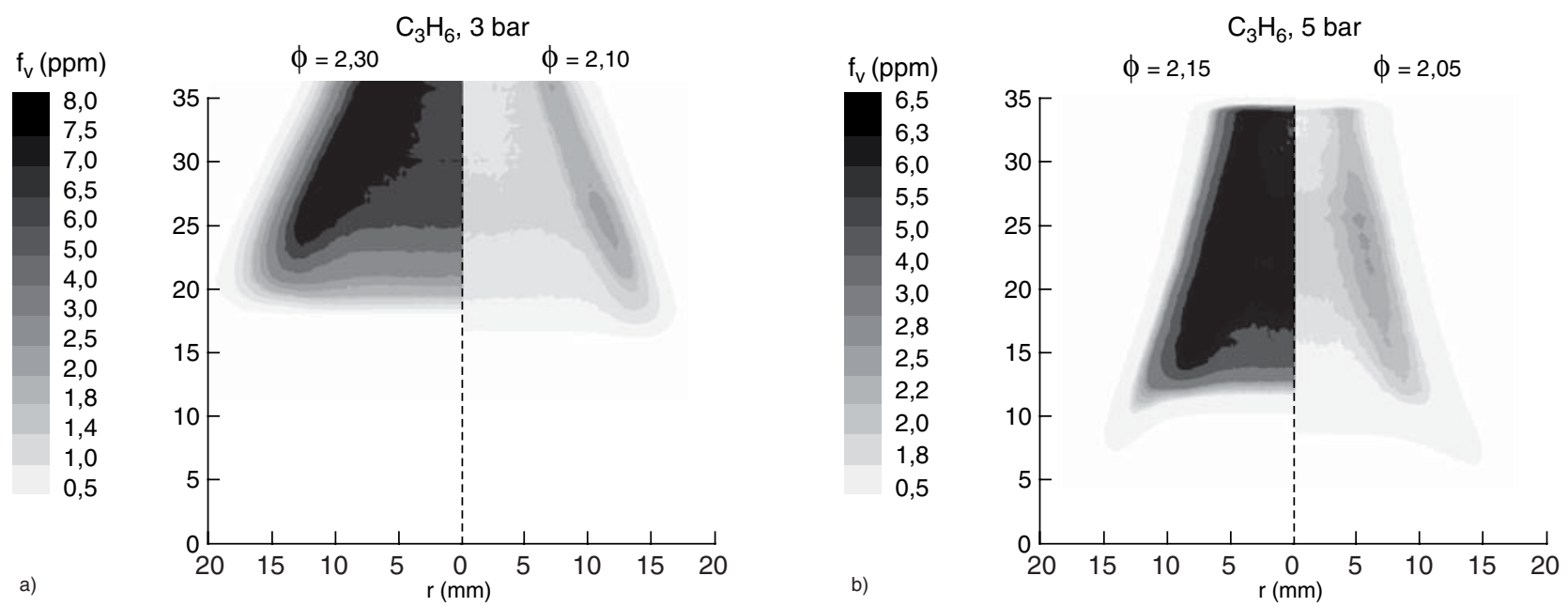

Figure 14

Distribution spatiale de $f_{v}$ (en ppm) reconstituée à partir de l'image IIL-2D. Spatial distribution of $f_{v}$ (in ppm) reconstructed from the $2 D$ LII image.

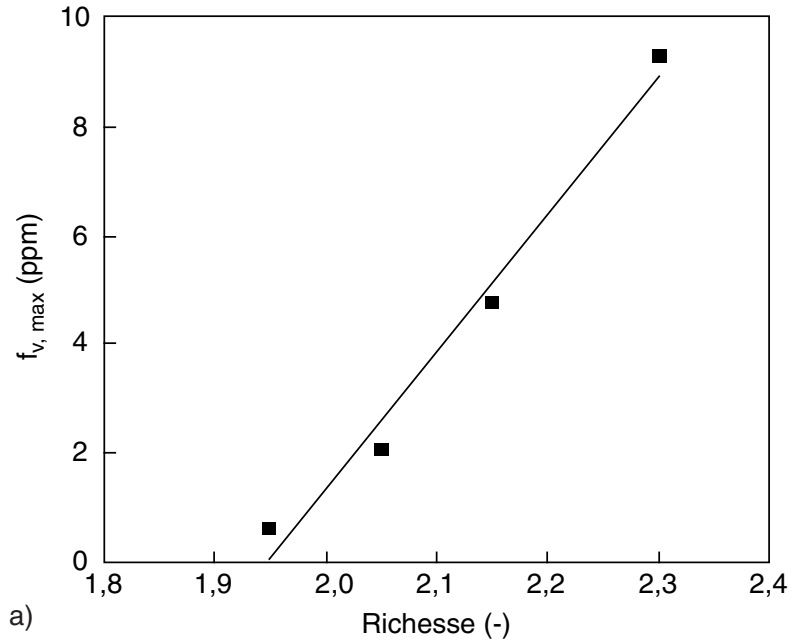

Figure 15a

Variation de $f_{v, \max }$ en fonction de $\phi$ pour une flamme $\mathrm{C}_{3} \mathrm{H}_{6}$ à une pression de 5 bar.

Variation of $f_{v, \text { max }}$ as a function of $\phi$ for $C_{3} H_{6}$ flames at a pressure of 5 bar.

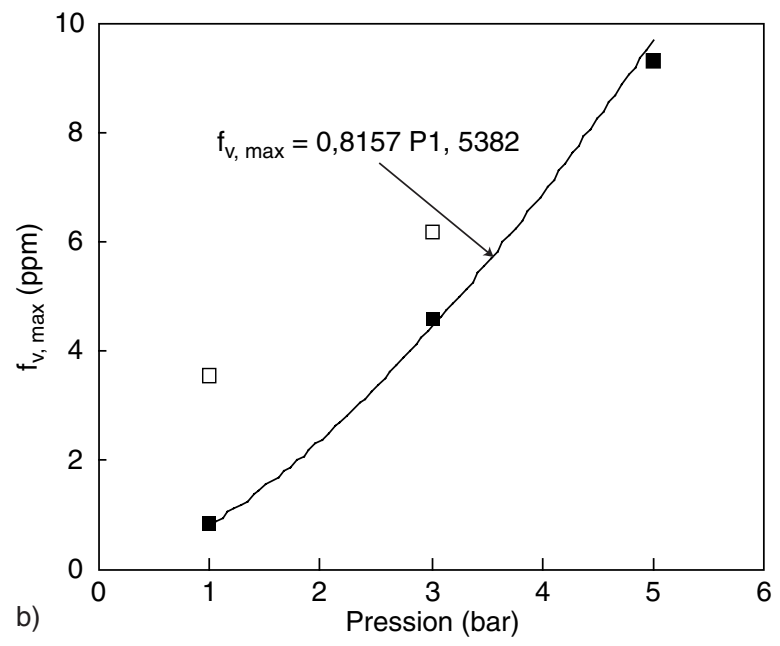

Figure 15b

Variation de $f_{v, \max }$ en fonction de la pression pour une flamme $\mathrm{C}_{3} \mathrm{H}_{6}$ à $\phi=2.30$.

Variation of $f_{v, \text { max }}$ as a function of pressure for $\mathrm{C}_{3} \mathrm{H}_{6}$ flames at $\phi=2.30$.
Raman anti-Stokes cohérente (DRASC). Cette valeur introduite dans un modèle théorique de refroidissement de la particule a permis d'évaluer le diamètre moyen des particules par la mesure du taux de décroissance du signal IIL(t) dans la flamme atmosphérique. Pour cette flamme, la dépendance monotone du taux de croissance de la concentration de la suie uniquement avec la température maximale a été confirmée. La valeur finale de la fraction volumique de la suie est proportionnelle à la richesse pour la flamme à 5 bar et croit non linéairement $\left(f_{v, \text { max }} \propto P^{1.5}\right)$ en fonction de la pression. La mesure de la taille des particules de suie a été limitée à la flamme atmosphérique par manque de validation expérimentale des constantes présentes dans les modèles théoriques de vaporisation de la suie à haute pression [46, 47].

\section{REMERCIEMENTS}

Les auteurs remercient chaleureusement HelmholtzGemeinschaft deutscher Forschungszentren pour le support financier (Programme PAZI) et la fondation Alexander von Humboldt pour la bourse de recherche octroyée à R. Hadef. 


\section{REFERENCES}

1 Kohse-Höinghaus K. and Jeffries J.B. (2002) Applied Combustion Diagnostics, Ed. Taylor \& Francis, New York.

2 Bockhorn H. (1994) Soot Formation in Combustion Mechanism and Models, Ed. Springer-Verlag, Berlin, Heidelberg.

3 Hansen J. and Nazarenko L. (2004) Soot climate forcing via snow and ice albedos, Proc. Natl. Acad. Sci. USA 101, 423 428.

4 Pope III C.A., Burnet R.T., Thun M.J., Calle E.E., Krewski D., Ito K. and Thurston G.D. (2002) Lung cancer, cardiopulmonary mortality, and long-term exposure to fine particulate air pollution, JAMA-J. Am. Med. Assoc. 6, 1132-1141.

5 Melton L.A. (1984) Soot diagnostics based on laser heating, Appl. Optics 23, 2201-2208.

6 Westbrook C.K. and Dryer F.L. (1984) Chemical kinetic modeling of hydrocarbon combustion, Prog. Energ. Combust. 10, 1-57.

7 Leung K.M. and Lindstedt R.P. (1995) Detailed kinetic modeling of $\mathrm{C}_{1}-\mathrm{C}_{3}$ alkane diffusion flames, Combust. Flame 102, 129-160.

8 Davis S.G. and Law C.K. (1998) Laminar flame speed and oxidation kinetics of iso-octane/air and $\mathrm{n} / \mathrm{heptane} /$ air flames, Proc. Comb. Inst. 27, 521-528.

9 Böhm H., Lamprecht A., Atakan B. and Kohse-Höinghaus K. (2000) Modelling of a fuel-rich premixed propene-oxygeneargon flame and comparison with experiments, Phys. Chem. Phys. 2, 4956-4961.

10 Schneider-Kühnle Y. (2004) Experimentelle Untersuchung rußender Hochdruckflammen mit laserdiagnostischen Messmethoden, PhD Thesis, Stuttgart.

11 Will S., Schraml S. and Leipertz A. (1995) Two-dimensional soot-particles sizing by time-resolved laser induced incandescence, Opt. Lett. 20, 2342-2344.

12 Vander Wal R., Ticich T.M. and Stephens A.B. (1999) Can soot primary particle size be determined using laser-induced incandescence, Combust. Flame 116, 291-296.

13 Mewes B. and Seitzmann J.M. (1997) Soot volume fraction and. particle size measurements with laser induced incandescence, Appl. Optics 36, 709-730.

14 Boiarcius C., Foucher F., Moreau B., Pajot O. and Mounaïm-Rousselle C. (2004) Simultaneous spatial and temporal resolved Laser-induced incandescence to study the soot particles formation, Proc. 12th International Symposium on Applications of Laser Techniques to Fluid Mechanics, Lisbon, Portugal.

15 Liu F., Smallwood G.J. and Snelling D.R. (2004) Effects of primary particle diameter and aggregate size distribution on the temperature of soot particles heated by pulsed lasers, $J$. Quant. Spectrosc. Ra. 93, 301-312.

16 Krüger V., Wahl C., Hadef R., Geigle K.P., Stricker W. and Aigner M. (2005) Comparison of laser-induced incandescence method with scanning mobility particle sizer technique: the influence of probe sampling and laser heating to soot particle size distribution, J. Meas. Sci. Technol. 16, 1477-1486.

17 Braun-Unkhoff M., Chrysostomou A., Frank P., Gutheil E., Lückerath R. and Stricker W. (1998) Experimental and numerical study on soot formation in laminar high-pressure flames, Proc. Combust. Inst. 27, 1565-1572.

18 Goeken D. and Johnson O. (2000) Runge-Kutta with higher order derivative approximations, Appl. Numer. Math. 34, 207-218.
19 Santoro R.J. and Shaddix C.R. (2002) Laser-induced incandescence, in Applied Combustion Diagnostics: K. KohseHöinghaus, J.B. Jeffries (Eds.), Taylor \& Francis, New York, 252-286.

20 Bouchardy P., Collin G., Magre P. and Péalat M. (1993) Thermométrie DRASC appliquée aux milieux en combustion, Oil Gas Sci. Technol. 48, 247-259.

21 Bengtsson P.E, Aldén M., Kröll S. and Nilsson D. (1990) Vibrational CARS thermometry in sooty flames: quantitative evaluation of $\mathrm{C}_{2}$ absorption interference, Combust. Flame 82, 199-210.

22 Geigle K.P., Schneider-Kühnle Y., Tsurikov M.S., Hadef R., Lückerath R., Krüger V., Stricker W. and Aigner M. (2005) Investigation of laminar pressurized flames for soot model validation using SV-CARS and LII, Proc. Combust. Inst. 30, $1645-1653$

23 Glassman I. (1988), Soot formation in combustion processes, Proc. Combust. Inst. 22, 295-311.

24 Melton T.R., Inal F., Senkan S.M. (2000) The effects of equivalence ratio on the formation of polycyclic aromatic hydrocarbons and soot in premixed ethane flames, Combust. Flame 121, 671-678.

25 Frenklach M., Clary D.W., Gardiner W.C. and Stein S.E. (1986) Effect of fuel structure on pathways to soot, Proc. Combust. Inst. 21, 1067-1076.

26 Woods L.T. and Haynes B.S. (1991) Soot surface growth at active sites, Combust. Flame 85, 523-525.

27 Frenklach M. (1996) On surface growth mechanism of soot particles, Proc. Combust. Inst. 26, 2285-2293.

28 Mauss F., Schäfer T. and Bockhorn H. (1994) Inception and growth of soot particles in dependence on the surrounding gas phase, Combust. Flame 99, 697-705.

29 Choi M.Y. and Jensen K.A. (1998), Calibration and correction of laser-induced incandescence for soot volume fraction measurements, Combust. Flame 112, 485-491.

30 Snelling D.R., Liu F., Smallwood G.J. and Gülder Ö.L. (2000) Evaluation of the nanoscale heat and mass transfer model of LII: prediction of the excitation intensity, Proc. 34th National Heat Transfer Conference, paper NHTC200012132.

31 Smallwood G.J., Snelling D.R., Liu F. and Gülder Ö.L. (2001) Clouds over soot evaporation: errors in modelling laser induced incandescence of soot, J. Heat Transf. ASME 123, 814-818.

32 Snelling D.R., Liu F., Smallwood G.J. and Gülder Ö.L. (2004) Determination of the soot absorption function and thermal accommodation coefficient using low-fluence LII in a laminar coflow ethylene diffusion flame, Combust. Flame 136, 180-190.

33 Michelsen H.A. (2003) Understanding and predicting the temporal response of laser-induced incandescence from carbonaceous particles, J. Chem. Phys. 118, 7012-7045.

34 Filippov A.V. and Rosner D.E. (2000) Energy transfer between an aerosol particle and gas at high temperature ratios in the Knudsen transition regime, Int. J. Heat Mass Transf. 43, 127-138.

35 Lehre T., Bockhorn H., Jungfleich B. and Schutz R. (2003) Development of a measuring technique for simultaneous in situ detection of nanoscaled particle size distributions and gas temperatures, Chemosphere 51, 1055-1066.

36 Lehre T., Jungfleisch B., Suntz R. and Bockhorn H. (2003) Size distributions of nanoscaled particles and gas temperatures from time-resolved laser-induced-incandescence measurements, Appl. Optics 42, 2021-2030. 
37 Schraml S., Dankers S., Bader K., Will S. and Leipertz A. (2000) Soot temperature measurements and implications for time-resolved laser-induced incandescence (TIRE-LII), Combust. Flame 120, 439-450.

38 Haynes B.S. and Wagner H.Gg. (1982) The surface growth phenomenon in soot formation, Z. Phys. Chem. 133, 201-213.

39 Mätzing H. and Wagner H.Gg. (1986) Proc. Comb. Inst. 21, $1047-1055$

40 Tsurikov M.S., Geigle K.P., Krüger V., Schneider-Kühnle Y., Stricker W., Lückerath R., Hadef R., Aigner M. (2005) Laser-based investigation of soot formation in laminar premixed flames at atmospheric and elevated pressures, Combust. Sci. Technol. 177-10, 1835-1862.

41 Böhm H., Feldermann Chr., Heidermann Th., Jander H., Lüers B. and Wagner H.Gg. (1992) Soot formation in premixed $\mathrm{C}_{2} \mathrm{H}_{4}$-air flames for pressures up to 100 bar, Proc. Combust. Inst. 24, 991-997.

42 Du J. and Axelbaum R.L. (1995) The effect of flame structure on soot-particle inception in diffusion flames, Combust. Flame 100, 367-375.
43 Maricq M., Harris S.J. and Szente J. (2003) Soot size distributions in rich premixed ethylene flames, Combust. Flame 132, 328-342

44 Bönig M., Feldermann Chr., Jander H., Lüers B., Rudolph G. and Wagner H.Gg. (1990) Soot formation in premixed $\mathrm{C}_{2} \mathrm{H}_{4}$ flat flames at elevated pressure, Proc. Combust. Inst. 23, 871878.

45 Miller I.M. and Maahs H.G. (1997) High pressure flame system for pollution studies with results for methane diffusion flames, NASA TN D 8407.

46 Kock B.F. and Roth P. (2003) Two-Color TR-LII Applied to In-Cylinder Diesel Particle Sizing, European Combustion Meeting, Orléans, France.

47 Hofmann M., Bessler W.G., Schulz C. and Jander H. (2003) Laser-induced incandescence for soot diagnostics at high pressures, Appl. Optics 42, 2052-2062.

Manuscrit final reçu en février 2006

Copyright $(\mathbb{C} 2006$ Institut français du pétrole

Permission to make digital or hard copies of part or all of this work for personal or classroom use is granted without fee provided that copies are not made or distributed for profit or commercial advantage and that copies bear this notice and the full citation on the first page. Copyrights for components of this work owned by others than IFP must be honored. Abstracting with credit is permitted. To copy otherwise, to republish, to post on servers, or to redistribute to lists, requires prior specific permission and/or a fee: Request permission from Documentation, Institut français du pétrole, fax. +33147527078 , or revueogst@ifp.fr. 